\title{
BMJ
}

\section{Toothbrushing, inflammation, and risk of cardiovascular disease: results from Scottish Health Survey}

\author{
Cesar de Oliveira, research fellow in epidemiology and public health, Richard Watt, professor and honorary \\ consultant in dental public health, Mark Hamer, senior research fellow in epidemiology and public health
}

Department of Epidemiology and Public Health, University College London, London WC1E 6BT Correspondence to: $\mathrm{R}$ Watt r.watt@ucl.ac.uk

Cite this as: BMJ 2010;340:c2451 doi:10.1136/bmi.c2451

\section{ABSTRACT}

Objective To examine if self reported toothbrushing behaviour is associated with cardiovascular disease and markers of inflammation ( $C$ reactive protein) and coagulation (fibrinogen).

Design National population based survey.

inflammation and chronic infections, including oral infections such as periodontal disease, have been investigated to explain the relation between dental disease and cardiovascular disease.

Poor oral hygiene is the major cause of periodontal disease, a chronic infection of the tissue surrounding
Setting Scottish Health Survey, which draws a nationally representative sample of the general population living in households in Scotland.

Participants 11869 men and women, mean age 50 (SD 11).

Main outcome measures Oral hygiene assessed from self reported frequency of toothbrushing. Surveys were linked prospectively to clinical hospital records, and Cox proportional hazards models were used to estimate the risk of cardiovascular disease events or death according to oral hygiene. The association between oral hygiene and inflammatory markers and coagulation was examined in a subsample of participants $(n=4830)$ by using general linear models with adjustments.

Results There were a total of 555 cardiovascular disease events over an average of 8.1 (SD 3.4) years of follow-up, of which 170 were fatal. In about $74 \%$ (411) of cardiovascular disease events the principal diagnosis was coronary heart disease. Participants who reported poor oral hygiene (never/rarely brushed their teeth) had an increased risk of a cardiovascular disease event (hazard ratio 1.7, 95\% confidence interval 1.3 to 2.3; P 0.001$)$ in a fully adjusted model. They also had increased concentrations of both $C$ reactive protein $(\beta$ $0.04,0.01$ to 0.08$)$ and fibrinogen $(0.08,-0.01$ to 0.18$)$. Conclusions Poor oral hygiene is associated with higher levels of risk of cardiovascular disease and low grade inflammation, though the causal nature of the association is yet to be determined.

\section{INTRODUCTION}

Over the past two decades, there has been an increasing interest in the possible link between dental disease, specifically periodontal disease, and cardiovascular disease. ${ }^{1}$ Inflammation plays an important role in the pathogenesis of atherosclerosis, and markers of low grade inflammation have been consistently associated with a higher risk of cardiovascular disease. ${ }^{2}$ Consequently, contributing factors associated with the teeth. It is one of the most common chronic infections and is associated with a moderate systemic inflammatory response, ${ }^{3}$ such as raised concentrations of $\mathrm{C}$ reactive protein and other inflammatory biomarkers. ${ }^{4-7}$ Systemic inflammation could represent the underlying mechanism that links oral health and cardiovascular disease. Thus, oral infections might add to the inflammatory burden of the individual and result in increased levels of cardiovascular risk based on serum $\mathrm{C}$ reactive protein and fibrinogen concentrations as a consequence of the systemic inflammatory response subsequent to low grade chronic infections. ${ }^{8}$ $\mathrm{C}$ reactive protein and fibrinogen are sensitive markers used to evaluate the inflammatory status of an individual, and the results of prospective longitudinal studies indicate that these markers might be useful predictors for future cardiovascular events in various populations. ${ }^{9}{ }^{10}$ Preliminary intervention trials showed a significant effect of treatment with systemic antibiotics in reducing systemic inflammatory markers. $^{1112}$

Previous smaller epidemiological studies that have examined the association between oral health and cardiovascular disease have generally used clinical assessments to identify periodontal disease. Such assessments might not be feasible in large scale population studies. Self reported measures of oral hygiene have been associated with clinically confirmed periodontal disease. ${ }^{13}$ The association between self reported oral hygiene behaviour and incident cardiovascular disease has not been previously examined in a large population study. We investigated whether a single item self reported measure of frequency of toothbrushing (as a proxy of periodontal disease) was associated with risk of cardiovascular disease events in a sample of adults from the Scottish Health Survey. In addition, we examined the association between frequency of toothbrushing and inflammatory markers $(\mathrm{C}$ reactive protein and fibrinogen) in a subset of participants. 
Table 1|Characteristics of study population in relation to oral hygiene. Figures are numbers (percentage) unless stated otherwise

\begin{tabular}{|c|c|c|c|c|}
\hline \multirow[b]{2}{*}{ Variable } & \multicolumn{3}{|c|}{ Frequency of toothbrushing } & \multirow[b]{2}{*}{$\begin{array}{l}P \text { value for } \\
\text { trend }\end{array}$} \\
\hline & $\begin{array}{c}\text { Twice a day } \\
(n=8481)\end{array}$ & $\begin{array}{l}\text { Once a day } \\
(n=2850)\end{array}$ & $\begin{array}{l}\text { Rarely/never } \\
\quad(n=538)\end{array}$ & \\
\hline Mean (SD) age (years) & $49.4(10.9)$ & $51.4(11.4)$ & $50.6(10.4)$ & $<0.001$ \\
\hline Men & $3265(38.5)$ & $1744(61.2)$ & $460(85.5)$ & $<0.001$ \\
\hline $\begin{array}{l}\text { Professional/intermediate } \\
\text { socioeconomic group }\end{array}$ & $3146(37.1)$ & $784(27.5)$ & $89(16.5)$ & $<0.001$ \\
\hline Dental visits (rarely) & $1382(16.3)$ & $920(32.3)$ & $336(62.5)$ & $<0.001$ \\
\hline Smokers & $2188(25.8)$ & $889(31.2)$ & $260(48.5)$ & $<0.001$ \\
\hline $\begin{array}{l}\text { Physical activity (bottom } \\
\text { third) }\end{array}$ & $2790(32.9)$ & $1134(39.8)$ & $248(46.1)$ & $<0.001$ \\
\hline Obesity* & 1602 (18.9) & $778(27.3)$ & $182(33.8)$ & $<0.001$ \\
\hline Hypertension† & $1848(21.8)$ & 789 (27.7) & $170(31.6)$ & $<0.001$ \\
\hline Diabetes & $186(2.2)$ & $91(3.2)$ & $36(6.7)$ & $<0.001$ \\
\hline
\end{tabular}

${ }^{*} \mathrm{BMI} \geq 30$.

†Defined from self reported doctor's diagnosis or clinic blood pressure reading $>140 / 90 \mathrm{~mm} \mathrm{Hg}$.

\section{METHODS}

Study design and participants

The Scottish Health Survey is a cross sectional survey (typically every three to five years) that draws a nationally representative sample of the general population living in Scottish households. For the present analysis we combined data from the 1995, 1998, and 2003 surveys in adults aged 35 and older as previously described elsewhere. ${ }^{14}$

\section{Assessment of oral health behaviours and covariates}

Survey interviewers visited eligible households and collected data on demographics and health behaviours (such as smoking, physical activity, and oral health behaviour) and measured height and weight. Physical activity interviews inquired about activity in the four weeks before the interview (1998 and 2003) or during a typical week (1995). Frequency of participation (for at least 20 minutes per occasion) was assessed across three domains of activity: leisure time sports (such as cycling, swimming, running, aerobics, dancing, and ball sports such as football and tennis); walking for any purpose; and domestic physical work (such as heavy housework, home improvement activities, manual and gardening work). The total physical activity frequency score was converted into sex specific thirds of weekly activity episodes. Oral health behaviour was assessed in all survey years from self reported frequency of visits to a dentist (at least once every six months, every one to two years, or rarely/never), and toothbrushing (brushing teeth twice a day, once a day, less than once a day). On a separate visit nurses collected information on medical history and family history of cardiovascular disease, blood pressure, and blood samples from consenting adults. The definition of hypertension was based on a self reported diagnosis from a doctor or a clinic blood pressure reading of $>140 / 90 \mathrm{~mm}$ Hg. Diabetes was based on a doctor's diagnosis. The covariables included in the present study were selected in a pre-specified protocol because they are all well established risk factors for cardiovascular disease. ${ }^{1516}$ Detailed information on the survey methods can be found elsewhere. ${ }^{17}$

\section{Follow-up for clinical events}

The surveys were linked to a database of hospital admissions and deaths in patients with follow-up until December 2007 (Information Services Division (ISD), Edinburgh). The database has been shown to be $94 \%$ accurate and 99\% complete when samples of computerised records for cardiovascular disease from the database were compared with the original case notes from patients. ${ }^{18}$ Information on deaths was ascertained from the general registrar office for Scotland. Classification of the underlying cause of death is based on information collected on cause of death from the medical certificate together with any additional information provided subsequently by the certifying doctor.

Our primary end point was a composite of fatal and non-fatal cardiovascular disease events. Mortality from cardiovascular causes was coded according to ICD-9 (international classification of diseases, ninth revision) codes 390-459 and ICD-10 (10th revision) codes I01-I99, and non-fatal events included hospital admissions related to cardiovascular disease, incorporating acute myocardial infarction, coronary artery bypass surgery, percutaneous coronary angioplasty, stroke, and heart failure.

\section{Blood analyses}

In a subsample of 4830 participants we collected peripheral blood samples in citrate and serum tubes for the assessment of $\mathrm{C}$ reactive protein and fibrinogen. This was optional, and several participants did not consent to having blood taken. Participants who did not consent to blood had poorer health risk profiles with higher body mass index (BMI) and a higher prevalence of hypertension, cardiovascular disease, and smoking. All blood samples were frozen at $-70^{\circ} \mathrm{C}$ until assay. The analysis of $\mathrm{C}$ reactive protein concentrations from serum was performed with the $\mathrm{N}$ Latex high sensitivity $\mathrm{C}$ reactive protein mono-immunoassay on a Behring Nephelometer II analyser. The limit of detection was $0.17 \mathrm{mg} / \mathrm{l}$, and the coefficient of variation was less than $6 \%$ for this assay. Fibrinogen concentrations were determined with the Organon Teknika MDA 180 analyser, with a modification of the Clauss thrombin clotting method, with a coefficient of variation of less than $10 \%$. All analyses were carried out in the same laboratory according to standard operating procedures by state registered medical laboratory scientific officers.

\section{Statistical analysis}

Cox proportional hazards models were used, with months as the time scale, to estimate the risk of cardiovascular disease events or death according to oral hygiene. The data were censored to December 2007 in event-free survivors. Initially, toothbrushing habit was included in the Cox model as a covariate, with 
adjustment for age and sex. In further multivariate models we adjusted for socioeconomic group using the registrar general classification (professional/intermediate, skilled non-manual, skilled manual, part skilled/unskilled), smoking (never, ex-smoker, current smoker), sex specific thirds of physical activity, frequency of dentist visits (at least once every six months, once every one to two years, rarely or never), BMI (underweight $<18.5$, normal weight 18.5-25.0, overweight 25.01-30.0, obese 30.1-40.0, morbidly obese $>40.0$ ), and family history of cardiovascular disease, hypertension, and diabetes. The proportional hazards assumption was examined by comparing the cumulative hazard plots grouped on exposure, although no violations were noted.

We examined the association between frequency of toothbrushing and inflammatory markers ( $\mathrm{C}$ reactive protein and fibrinogen) using general linear models with adjustments for age, sex, socioeconomic group, smoking, visits to dentist, BMI, family history of cardiovascular disease, hypertension, and diabetes, and acute infections (including influenza, pneumonia, bronchitis, and upper respiratory tract infections in the three weeks before assessment). C reactive protein was $\log$ transformed to normalise the data. All analyses were performed with SPSS (version 14), and all tests of significance were based on two sided probability.

\section{RESULTS}

We removed from the analyses 3685 participants who were edentulous (no natural teeth) and 386 with existing cardiovascular disease. Participants with missing demographic data $(\mathrm{n}=204)$ were also excluded, leaving a final sample size of 11869 (46.1\% men, mean age 50.0 (SD 11.0)). The excluded edentate participants were older and more likely to be women and smokers.

Oral health behaviour was generally good, with about $62 \%$ (14718) of participants reporting regular (at least every six months) visits to a dentist and 71\% (8481) reporting good oral hygiene (brushing teeth twice a day). Participants who brushed their teeth less often than twice a day were slightly older, more likely to be men, and of lower social status and had a high prevalence of risk factors including smoking, physical inactivity, obesity, hypertension, and diabetes (table 1).

Table 2 |Cox regression models for toothbrushing and cardiovascular disease (CVD) events (fatal and non-fatal combined)

\begin{tabular}{lcccc} 
& & \multicolumn{3}{c}{ Hazard ratio (95\% CI) } \\
\cline { 2 - 5 } Toothbrushing & $\begin{array}{c}\text { CVD } \\
\text { events/total }\end{array}$ & $\begin{array}{c}\text { Adjusted } \\
\text { for age/sex }\end{array}$ & Model 1 $^{*}$ & Model 2 $\dagger$ \\
Total & $555 / 11869$ & - & - & - \\
\hline Twice a day & $308 / 8481$ & 1.0 (reference) & 1.0 (reference) & 1.0 (reference) \\
\hline Once a day & $188 / 2850$ & $1.4(1.2$ to 1.7$)$ & $1.3(1.1$ to 1.5$)$ & 1.3 (1.0 to 1.5$)$ \\
\hline SOnce a day & $59 / 538$ & $2.3(1.8$ to 3.1$)$ & $1.8(1.3$ to 2.4$)$ & 1.7 (1.3 to 2.3) \\
\hline P value for trend & - & 0.001 & 0.001 & 0.001
\end{tabular}

*Adjusted for age, sex, socioeconomic group, smoking, physical activity, and visits to dentist.

†Additionally adjusted for BMI, family history of cardiovascular disease, hypertension (diagnosed by doctor or clinic blood pressure $\$ 140 / 90 \mathrm{~mm} \mathrm{Hg}$ ), diabetes diagnosed by doctor.
Participants from the different survey years were comparable in terms of demographics and risk factors.

There were 555 cardiovascular disease events over an average of 8.1 (SD 3.4) years of follow-up, of which 170 were fatal. In about 74\% (411) of cardiovascular disease events the principal diagnosis was coronary heart disease. The mean age of event-free survivors compared with participants with a recorded cardiovascular disease event was 49.6 (SD 10.9) and 57.0 (SD 10.3), respectively ( $\mathrm{P}<0.001)$, at baseline. In age and sex adjusted analyses participants reporting poor dental hygiene had an increased risk of cardiovascular disease events and cardiovascular disease death (tables 2 and 3). In further multivariate models the associations were attenuated, although they remained significant in the case of cardiovascular disease events. Participants who reported less frequent toothbrushing had a $70 \%$ increased risk of a cardiovascular disease event in fully adjusted models compared with participants who brushed their teeth twice a day. The other independent predictors of cardiovascular disease events included smoking (hazard ratio $2.4,95 \%$ confidence interval 1.9 to 2.9$)$, hypertension $(1.7,1.4$ to 2.0 ), and diabetes $(1.9,1.4$ to 2.7$)$.

As some previous studies have suggested effect modification by age, sex, and smoking status in relation to oral health and cardiovascular disease, ${ }^{19}$ we performed various sensitivity analyses. There were no clear sex differences in our results; the age adjusted hazard ratio for cardiovascular disease events in relation to toothbrushing less than once a day was 2.2 (1.6 to 3.1) in men and 3.6 (1.7 to 7.7) in women. There was also no difference by age; the age/sex adjusted hazard ratio was 2.7 (1.4 to 5.4) in participants aged 65 or above compared with 2.2 (1.6 to 3.0) in those aged less than 65 . There were no differences between smokers (2.0, 1.3 to 3.0$)$ and those who had never smoked (2.0, 1.1 to 3.6).

There were significant associations between frequency of toothbrushing and markers of low grade systemic inflammation (table 4). Participants who brushed their teeth less often had increased concentrations of both $\mathrm{C}$ reactive protein and fibrinogen. These associations remained significant after multiple adjustments. In the subsample of participants with available biological data we re-ran the survival analyses to examine if the association between toothbrushing and cardiovascular disease was weakened by the inclusion of inflammatory markers (see appendix on bmj.com). In these analyses there were 161 cardiovascular disease events and the age and sex adjusted associations were similar compared with the main sample. The introduction of $\mathrm{C}$ reactive protein and fibrinogen did partly attenuate the point estimates, thus suggesting a possible mediating role.

\section{DISCUSSION}

Toothbrushing is associated with cardiovascular disease, even after adjustment for age, sex, socioeconomic group, smoking, visits to dentist, BMI, family history of cardiovascular disease, hypertension, and diagnosis of 
Table 3 |Cox regression models for toothbrushing and death from cardiovascular disease (CVD)

\begin{tabular}{|c|c|c|c|c|}
\hline \multirow[b]{2}{*}{ Toothbrushing } & \multirow[b]{2}{*}{$\begin{array}{c}\text { Fatal CVD } \\
\text { events/total }\end{array}$} & \multicolumn{3}{|c|}{ Hazard ratio $(95 \% \mathrm{Cl})$} \\
\hline & & $\begin{array}{c}\text { Adjusted } \\
\text { for age/sex }\end{array}$ & Model 1* & Model $2 \dagger$ \\
\hline Total & $170 / 11869$ & - & - & - \\
\hline Twice a day & $97 / 8481$ & 1.0 (reference) & 1.0 (reference) & 1.0 (reference) \\
\hline Once a day & $54 / 2850$ & $1.2(0.8$ to 1.8$)$ & $1.1(0.8$ to 1.5$)$ & 1.1 (0.8 to 1.5$)$ \\
\hline ¿Once a day & $19 / 538$ & $2.4(1.5$ to 4.0$)$ & $1.6(1.0$ to 2.8$)$ & 1.5 (0.9 to 2.6$)$ \\
\hline$P$ value for trend & - & 0.003 & 0.194 & 0.345 \\
\hline
\end{tabular}

*Adjusted for age, sex, socioeconomic group, smoking, physical activity, and visits to dentist.

†Additionally adjusted for BMI, family history of cardiovascular disease, hypertension (diagnosed by doctor or clinic blood pressure $>140 / 90 \mathrm{~mm} \mathrm{Hg}$ ), diabetes diagnosed by doctor.

diabetes. Our results largely confirm those of previous studies. ${ }^{2021}$ We examined the association between toothbrushing behaviour and cardiovascular disease and whether markers of low grade inflammation/coagulation were associated with low frequency of toothbrushing. Our results also suggest that toothbrushing is associated with concentrations of $\mathrm{C}$ reactive protein and fibrinogen. To the best of our knowledge, this is the first study to show an association between a single item self reported measure of toothbrushing and incident cardiovascular disease in a large representative sample of adults without overt cardiovascular disease. As self reported measures of oral hygiene have been associated with clinically confirmed periodontal disease,${ }^{13}$ a simple self report measure of toothbrushing could therefore be associated with future risk for cardiovascular disease.

\section{Oral health and cardiovascular disease}

The role of oral health in the aetiology of cardiovascular disease has received considerable attention. Periodontal disease is a complex chronic inflammatory disease, resulting in a loss of connective tissue and bone support of the teeth. ${ }^{22}$ It is a major cause of tooth loss in adults aged over 40, and, according to the World Health Organization, affects people worldwide at prevalence rates of up to $10-20 \%$ for the most severe forms. ${ }^{23}$ Periodontal disease is highly prevalent, especially in late middle age when coronary artery disease is also most common, ${ }^{24}$ and it is caused mostly by poor oral hygiene.

In our study, participants who brushed their teeth less often had a 70\% increased risk of a cardiovascular disease event in fully adjusted models. These results confirm findings from several observational epidemiological studies that showed that poor periodontal health status is associated with an increased risk of cardiovascular disease. ${ }^{1}$ In a study of 15 year followup data from the First National Health and Nutritional Examination Survey (NHANES I) Epidemiologic Follow-up Study, DeStefano et al found that people with periodontal disease had a $25 \%$ increased risk for coronary heart disease relative to those with minimal periodontal disease, after adjustment for age, sex, race, education, poverty index, marital status, systolic blood pressure, total cholesterol concentration, diabetes, BMI, and alcohol consumption. ${ }^{25}$ In a longitudinal study, Beck et al found that the odds ratios were 1.5 for total coronary heart disease and 1.9 for fatal coronary heart disease among people with periodontal bone loss compared with those without bone loss, after adjustment for several risk factors for cardiovascular disease. $^{26}$

One meta-analysis concluded that periodontal disease and poor oral health overall indeed contribute to the pathogenesis of cardiovascular disease. ${ }^{27}$ Another meta-analysis, by Bahekar et al, confirmed that having periodontal disease might enhance the risk for cardiovascular disease but concluded that this risk was not robust. $^{20}$

Periodontal disease seems to be associated with a $19 \%$ increase in the risk of future cardiovascular disease. This increase in relative risk is more prominent $(44 \%)$ in people aged under 65 . The increment of risk between people with or without periodontal disease in the general population is modest, at around 20\%, because nearly $40 \%$ of the population have periodontal disease. This modest increase might, however, have a profound public health impact. ${ }^{28}$

In our study, less frequent toothbrushing was associated with increased concentrations of both $\mathrm{C}$ reactive protein and fibrinogen, and these associations remained significant after multiple adjustments including acute infections such as influenza. The work on serum markers of inflammation in both cardiovascular and periodontal research is extensive. The literature clearly shows that raised pro-inflammatory cytokines are present in both cardiovascular disease and periodontal disease. As a result, accumulating evidence has associated severe periodontal disease with increased

Table $4 \mid$ Adjusted regression coefficients $(95 \% \mathrm{Cl})$ of toothbrushing on inflammatory markers $(\mathrm{n}=4830)$

\begin{tabular}{|c|c|c|c|c|c|c|}
\hline & \multicolumn{3}{|c|}{$\mathrm{C}$ reactive protein } & \multicolumn{3}{|c|}{ Fibrinogen } \\
\hline & Mean (SD) $\mathrm{mg} \backslash \mathrm{l}$ & $\underset{\beta^{\star}}{\text { Age/sex adjusted }}$ & Fully adjusted $\dagger \beta$ & Mean (SD) gll & Age/sex adjusted $\beta$ & Fully adjusted $\dagger \beta$ \\
\hline Twice a day & 3.07 (6.41) & Reference & Reference & $2.86(0.70)$ & Reference & Reference \\
\hline Once a day & $3.51(6.55)$ & 0.04 (0.02 to 0.06$)$ & $0.02(0.00$ to 0.04$)$ & $2.95(0.71)$ & 0.11 (0.06 to 0.15$)$ & $0.06(0.02$ to 0.11$)$ \\
\hline ¿Once a day & $4.18(6.95)$ & 0.09 (0.06 to 0.13$)$ & 0.04 (0.01 to 0.08$)$ & $2.98(0.77)$ & $0.22(0.12$ to 0.32$)$ & $0.08(-0.01$ to 0.18$)$ \\
\hline $\mathrm{P}$ value for trend & - & $<0.001$ & 0.046 & - & $<0.001$ & 0.015 \\
\hline
\end{tabular}

*Regression coefficients represent log transformed values.

$\dagger$ †djusted for age, sex, socioeconomic group, smoking, BMI, family history of cardiovascular disease, hypertension (diagnosed by doctor or clinic blood pressure $\$ 140 / 90 \mathrm{~mm} \mathrm{Hg}$ ), diabetes diagnosed by doctor, visits to dentist, acute infections (including influenza, pneumonia, bronchitis, and upper respiratory tract infections). 


\begin{tabular}{l}
\hline WHAT IS ALREADY KNOWN ON THIS TOPIC \\
\hline $\begin{array}{l}\text { There is a link between cardiovascular disease and } \\
\text { periodontal disease } \\
\text { Poor oral hygiene is associated with periodontal disease, } \\
\text { a common chronic inflammatory condition } \\
\text { WHAT THIS STUDY ADDS } \\
\text { These results confirm and further strengthen the suggested } \\
\text { association between oral hygiene and the risk of } \\
\text { cardiovascular disease } \\
\text { Inflammatory markers are significantly associated with poor } \\
\text { oral health behaviour }\end{array}$ \\
\hline
\end{tabular}

odds of future cardiovascular disease events. ${ }^{3}$ Our study suggests a possible role of poor oral hygiene in the risk of cardiovascular disease via systemic inflammation. Raised inflammatory and homoeostatic responses as well as lipid metabolism disturbance caused by periodontal infection might be possible pathways underlying the observed association between periodontal disease and the increased risk for cardiovascular disease. ${ }^{29}$ Few studies, however, have examined these potential pathways. If these biological mechanisms are responsible for a slight increase in the risk of cardiovascular disease, better controlled and larger studies will be needed to identify them. Such efforts would be important because of the relatively high prevalence of periodontal disease.

\section{Strengths and limitations}

The Scottish Health Survey is nationally representative, with a rigorous design and data linked to a patient based database of hospital admissions and deaths with follow-up. The Scottish population is relatively homogeneous, with a high incidence of cardiovascular disease and poor indicators of oral health, thus our findings have high relevance to this population.

Though clinical data regarding the periodontal disease status of the participants might have strengthened our findings, previous research has also shown a good correlation between self report and clinical evaluation of periodontal disease. ${ }^{13} \mathrm{We}$ had no follow-up data on toothbrushing behaviour. There is, however, evidence showing stability of oral health related behaviour such as toothbrushing and dental flossing, ${ }^{30}$ thus small changes in oral health behaviour are unlikely to affect the present findings. Both residual confounding and potential influence of effect modifiers could be responsible for a substantial attenuation of the relative risk in fully adjusted models. In addition, misclassification of both the exposure and the outcome could have played a role.

\section{Conclusions}

Our results confirmed and further strengthened the suggested association between oral hygiene and the risk of cardiovascular disease. Furthermore, inflammatory markers were significantly associated with poor oral health behaviour. Future experimental studies will be needed to confirm whether the observed association between oral health behaviour and cardiovascular disease is in fact causal or merely a risk marker. Nevertheless, use of a simple one item measure of self reported toothbrushing could be a useful and cost effective marker of future health risk in large scale population studies.

Given the high prevalence of oral infections in the population, doctors should be alert to the possible oral source of an increased inflammatory burden. In addition, educating patients in improving personal oral hygiene is beneficial to their oral health regardless of the relation with systemic disease.

Contributors: $\mathrm{CdeO}$ conceived and designed the study, interpreted the data, drafted the article, and is guarantor. MH analysed the data. RW and $\mathrm{MH}$ interpreted the data and revised the article. All authors approved the final version.

Funding: This research received no specific grant from any funding agency in the public, commercial, or not-for-profit sectors. $\mathrm{CdeO}$ is funded by the National Institute of Aging/National Institute of Health and British government departments. MH is funded by the British Heart Foundation. The Scottish Health Survey is funded by the Scottish Executive. The funders had no role in the present study.

Competing interests: All authors have completed the Unified Competing Interest form at www.icmje.org/coi_disclosure.pdf (available on request from the corresponding author) and declare (1) No financial support for the submitted work from anyone other than their employer; (2) No financial relationships with commercial entities that might have an interest in the submitted work; (3) No spouses, partners, or children with relationships with commercial entities that might have an interest in the submitted work; (4) No non-financial interests that may be relevant to the submitted work.

Ethical approval: This study was approved by the London research ethics council and informed consent was given by all participants.

Data sharing: The Scottish Health Survey dataset is openly available at the UK Data Archive (www.data-archive.ac.uk/). The follow-up data on hospital admissions can be made available on request from the Information Services Division, Edinburgh (www.isdscotland.org/isd/ 3348.html).

1 Persson GR, Persson RE. Cardiovascular disease and periodontitis: an update on the associations and risk. J Clin Periodontol 2008;35:362-79.

2 Danesh J, Wheeler JG, Hirschfield GM, Eda S, Eiriksdottir G, Rumley A, et al. C-reactive protein and other circulating markers of inflammation in the prediction of coronary heart disease. $N$ Engl J Med 2004;350:1387-97.

3 D'Aiuto F, Ready D, Tonetti MS. Periodontal disease and C-reactive protein-associated cardiovascular risk. J Periodont Res 2004;39:236-41.

4 Loos BG, Craandijk J, Hoek FJ, Wertheim-van Dillen PM, van der Velden U. Elevation of systemic markers related to cardiovascular diseases in the peripheral blood of periodontitis patients. J Periodontol 2000;71:1528-34.

5 Noack B, Genco RJ, Trevisan M, Grossi S, Zambon JJ, De Nardin E. Periodontal infections contribute to elevated systemic C-reactive protein level. J Periodontol 2001;72:1221-7.

6 Slade GD, Offenbacher S, Beck JD, Heiss G, Pankow JS. Acute-phase inflammatory response to periodontal disease in the US population.J Dent Res 2000;79:49-57.

7 Slade GD, Ghezzi EM, Heiss G, Beck JD, Riche E, Offenbacher S. Relationship between periodontal disease and C-reactive protein among adults in the atherosclerosis risk in communities study. Arch Intern Med 2003;163:1172-9.

8 Libby P, Ridker PM, Maseri A. Inflammation and atherosclerosis. Circulation 2002;105:1135-43.

9 Danesh J, Whincup P, Walker M, Lennon L, Thomson A, Appleby P, et al. Low grade inflammation and coronary heart disease: prospective study and updated meta-analyses. $B M$ J 2000;321:199-204.

10 Ridker PM, Cushman M, Stampfer MJ, Tracy RP, Hennekens CH. Inflammation, aspirin, and the risk of cardiovascular disease in apparently healthy men. N Engl J Med 1997;336:973-9.

11 Stone AF, Mendall MA, Kaski JC, Edger TM, Risley P, Poloniecki J, et al. Effect of treatment for Chlamydia pneumoniae and Helicobacter 
pylori on markers of inflammation and cardiac events in patients with acute coronary syndromes: South Thames Trial of Antibiotics in Myocardial Infarction and Unstable Angina (STAMINA). Circulation 2002;106:1219-23.

12 Wiesli P, Czerwenka W, Meniconi A, Maly FE, Hoffman U, Vetter W, et al. Roxithromycin treatment prevents progression of peripheral arterial occlusive disease in Chlamydia pneumoniae seropositive men: a randomized, double-blind, placebo-controlled trial. Circulation 2002;105:2646-52.

13 Miller K, Eke PI, Schoua-Glusberg A. Cognitive evaluation of selfreport questions for surveillance of periodontitis. J Periodontol 2007;78:1455-62.

14 Hamer M, Stamatakis E, Steptoe A. Psychiatric hospital admissions, behavioral risk factors, and all-cause mortality: the Scottish Health Survey. Arch Intern Med 2008;168:2474-9.

15 Danaei G, Ding EL, Mozaffarian D, Taylor B, Rehm J, Murray C), et al. The preventable causes of death in the United States: comparative risk assessment of dietary, lifestyle, and metabolic risk factors. PLoS Med 2009;6:e1000058.

16 Emberson JR, Whincup PH, Morris RW, Walker M. Re-assessing the contribution of serum total cholesterol, blood pressure and cigarette smoking to the aetiology of coronary heart disease: impact of regression dilution bias. Eur Heart J 2003;24:1719-26.

17 Scottish Government Statistics. Scottish Health Survey Publications www.scotland.gov.uk/Topics/Statistics/Browse/Health/scottishhealth-survey.

18 Information Services Division, NHS National Services Scotland. Information and statistics. 2010. www.isdscotland.org/isd/3348. html.

19 Desvarieux M, Schwahn C, Völzke H, Demmer RT, Ludemann J, Kessler C, et al. Gender differences in the relationship between periodontal disease, tooth loss, and atherosclerosis. Stroke 2004;35:2029-35.

20 Bahekar AA, Singh S, Saha S, Molnar J, Arora R. The prevalence and incidence of coronary heart disease is significantly increased in periodontitis: a meta-analysis. Am Heart J 2007;154:830-7.
21 Mustapha IZ, Debrey S, Oladubu M, Ugarte R. Cardiovascular disease markers of systemic bacterial exposure in periodontal disease and risk: a systematic review and meta-analysis. J Periodontol 2007;78:2289-302.

22 Pihlstrom BL, Michalowicz BS, Johnson NW. Periodontal diseases. Lancet 2005;366:1809-20.

23 Petersen PE. The World Oral Health Report 2003: continuous improvement of oral health in the 21st century-the approach of the WHO global oral health programme. Community Dent Oral Epidemiol 2003;31(suppl 1):3-23S.

24 De Nardin E. The role of inflammatory and immunological mediators in periodontitis and cardiovascular disease. Ann Periodontol 2001;6:30-40.

25 DeStefano F, Anda RF, Kahn HS, Williamson DF, Russell CM. Dental disease and risk of coronary heart disease and mortality. $B M$ J 1993;306:688-91.

26 Beck J, Garcia R, Heiss G, Vokonas PS, Offenbacher S. Periodontal disease and cardiovascular disease. J Periodontol 1996;67:1123-37.

27 Meurman $\mathrm{HH}$, Sanz M, Janket SJ. Oral health, atherosclerosis, and cardiovascular disease. Crit Rev Oral Biol Med 2004;15:403-13.

28 Janket SJ, Baird AE, Chuang SK, Jones JA. Meta-analysis of periodontal disease and risk of coronary heart disease and stroke. Oral Surg Oral Med Oral Pathol Oral Radiol Endod 2003;95:559-69.

29 Wu T, Trevisan M, Genco RJ, Falkner KL, Dorn JP, Sempos CT. Examination of the relation between periodontal health status and cardiovascular risk factors: serum total and high density lipoprotein cholesterol, C-reactive protein, and plasma fibrinogen. $\mathrm{Am}$ J Epidemiol 2000;151:273-82.

30 Astrøm AN. Stability of oral health-related behaviour in a Norwegian cohort between the ages of 15 and 23 years. Community Dent Oral Epidemiol 2004;32:354-62.

Accepted: 12 April 2010 